\title{
Role of disorder in half-filled high Landau levels
}

\author{
D. N. Sheng, ${ }^{1}$ Ziqiang Wang, ${ }^{2}$ and B. Friedman ${ }^{3}$ \\ ${ }^{1}$ Department of Physics and Astronomy, California State University, Northridge, California 91330 \\ ${ }^{2}$ Department of Physics, Boston College, Chestnut Hill, Massachusetts 02467 \\ ${ }^{3}$ Department of Physics, Sam Houston State University, Huntsville, Texas 77341-2267
}

(Received 30 August 2002; published 29 October 2002)

\begin{abstract}
We study the effects of disorder on the quantum Hall stripe phases in half-filled high Landau levels using exact numerical diagonalization. We show that, in the presence of weak disorder, a compressible, striped charge-density wave, becomes the true ground state. The projected electron-density profile resembles that of a smectic liquid. With increasing disorder strength $W$, we find that there exists a critical value, $W_{c}$ $\sim 0.12 e^{2} / \epsilon \ell$, where a transition/crossover to an isotropic phase with strong local electron-density fluctuations takes place. The many-body density of states are qualitatively distinguishable in these two phases and help elucidate the nature of the transition.
\end{abstract}

DOI: $10.1103 /$ PhysRevB.66.161103

PACS number(s): 73.43.-f, 73.40.Kp, 73.50.Jt

Recent experiments have discovered a set of compressible states with anisotropic low-temperature magnetotransport properties in high-mobility quantum Hall systems near half filling of the high Landau levels (LLs). ${ }^{1}$ Subsequently, it was proposed that these properties, emerging as a consequence of strong long-range electronic correlations and the quenching of the kinetic energy, are manifestations of the electronic liquid-crystal phases with partially broken translation and rotational symmetries. ${ }^{2,3}$ The anomalous anisotropic transport can be interpreted as arising from a rotational symmetry breaking ground state, i.e., a smectic or a stripe ordered charge-density wave (CDW) phase. $^{2-6}$ Remarkably, a unidirectional CDW state has been predicted to occur in half-filled high LLs in the Hartree-Fock (HF) theory. ${ }^{7}$ However, it has been known that the smectic liquid phase in the HF theory is always unstable to translation-symmetry breaking density modulations along the stripes. ${ }^{2-6}$ The latter produces an anisotropic but incompressible striped crystal that is insulating in both directions, inconsistent with experiments. It is thus important to consider quantum fluctuations beyond the $\mathrm{HF}$ theory, which is under active investigation.

In this paper, we study the effects of disorder on the ground state and low-energy excitations in half-filled high LLs using exact numerical diagonalizations of finite systems with up to twelve electrons. Previously, exact diagonalizations were carried out by Rezayi, Haldane, and Yang ${ }^{8}$ (hereafter referred to as RHY) for clean systems. Although the ground state in this case does not directly exhibit stripe order, RHY discovered that the static density response function $\chi(\mathbf{q})$ and the correlation function $S_{0}(\mathbf{q})$ are both sharply and strongly peaked at $\mathbf{q}^{*}=\left(q_{x}^{*}, 0\right)$, indicative of the tendency towards the formation of a unidirectional CDW. They conjectured that in the thermodynamic limit, an external modulation with a period matching $\mathbf{q}^{*}$ will result in a quantum Hall stripe phase. We will show that the presence of disorder leads to several interesting and important results for the ground state and the low-energy excitations.

(i) A very weak random potential associated with disorder induces a compressible quantum smectic phase as the true ground state. (ii) There exists a critical/characteristic disorder strength, $W_{c} \sim 0.12 e^{2} / \epsilon \ell$, where $\ell$ is the magnetic length, which marks a transition/crossover from the anisotropic smectic phase at $W<W_{c}$ to an isotropic fluid phase at $W>W_{c} .{ }^{9} \mathrm{We}$ illustrate the changes in the collective excitation spectrum in terms of the disorder-averaged many-body density of states (mDOS) at low energies. We show that the latter exhibits an intriguing crossover from that resembling a finite density of in-gap states in the smectic phase to that of a linearly vanishing behavior in the isotropic phase.

We consider a two-dimensional system in an $a \times b$ rectangular cell with periodic boundary conditions imposed in both $x$ and $y$ directions. ${ }^{10}$ In the presence of a strong magnetic field, one can project the Hamiltonian onto the uppermost, partially filled, $N$ th Landau level. The projected Hamiltonian in the presence of both Coulomb interaction and disorder can be written as

$$
\begin{aligned}
H= & \sum_{i<j} \sum_{\mathbf{q}} e^{-q^{2} / 2}\left[L_{N}\left(q^{2} / 2\right)\right]^{2} V(q) e^{i \mathbf{q} \cdot\left(\mathbf{R}_{i}-\mathbf{R}_{j}\right)} \\
& +\sum_{i} \sum_{\mathbf{q}} e^{-q^{2} / 4} L_{N}\left(q^{2} / 2\right) V_{\mathrm{imp}}(q) e^{i \mathbf{q} \cdot \mathbf{R}_{i}},
\end{aligned}
$$

where $\mathbf{R}_{i}$ is the guiding center coordinate of the $i$ th electron, ${ }^{8}$ $L_{N}(x)$ is the Laguerre polynomial, $V(q)=2 \pi e^{2} / \epsilon q$ is the Coulomb potential, and $V_{\text {imp }}(q)$ is the impurity potential. We set $\ell=1$ and $e^{2} / \epsilon \ell=1$ for convenience. In Eq. (1), the wave vector $\mathbf{q}=2 \pi\left(i_{x} / a, i_{y} / b\right)$ with $i_{x}$ and $i_{y}$ integers. The disorder potential is generated according to the correlation relation in $q$-space $\left\langle V_{\text {imp }}(q) V_{\text {imp }}\left(-q^{\prime}\right)\right\rangle=W^{2} \delta_{q,-q^{\prime}}$, which corresponds to $\left\langle V(\mathbf{r}) V\left(\mathbf{r}^{\prime}\right)\right\rangle=W^{2} \delta\left(\mathbf{r}-\mathbf{r}^{\prime}\right)$ in real space, where $W$ is the strength of the disorder in units of $e^{2} / \epsilon \ell$. We consider up to $N_{e}=12$ electrons, spanning a Hilbert space of size $N_{\text {basis }}=2,704,156$. Since the disorder potential spoils the symmetry classifications of the states used in RHY, we must deal with the entire Hilbert space. We obtain the exact lowenergy eigenstates and eigen-wave-functions using the Lanczos diagonalization method.

The most direct probe of charge-density order in the ground state is the projected electron density $\rho(\mathbf{r})$, i.e., the 

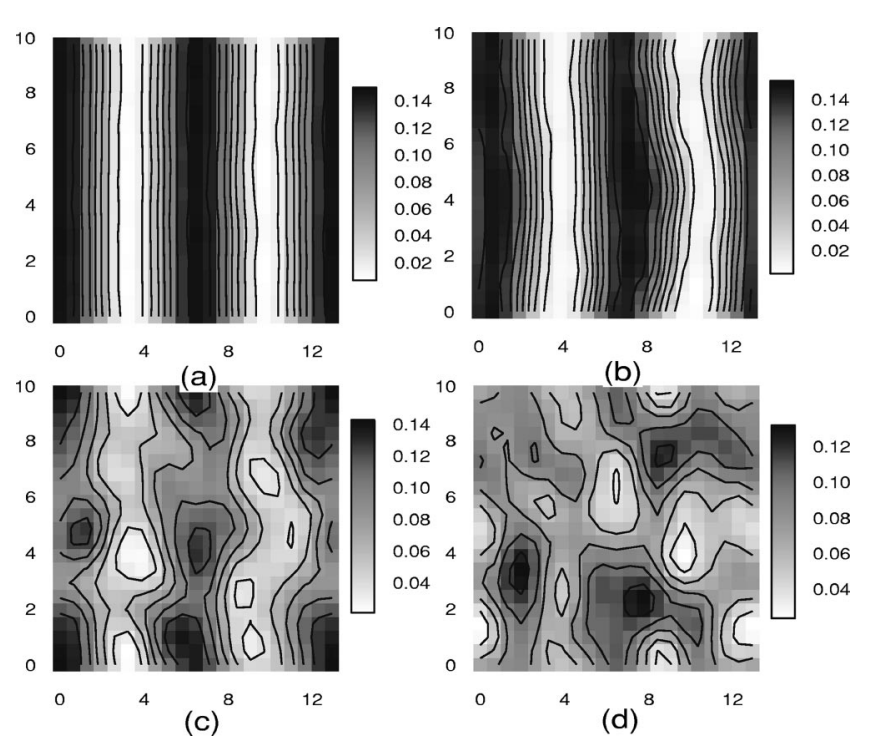

FIG. 1. The projected electron density $\rho(\mathbf{r})$ at four different disorder strengths for $N_{e}=10, N=2$, and aspect ratio $A=0.75$. (a) $W=0.015$, (b) $W=0.045$, (c) $W=0.10$, and (d) $W=0.14$.

equivalent electron density in the lowest LL describing the spatial distribution of the guiding center. ${ }^{8,11}$ It is given by

$$
\rho(\mathbf{r})=\sum_{j 1, j 2}\left\langle 0\left|a_{j_{1}}^{+} \phi_{j_{1}}^{*}(\mathbf{r}) \phi_{j_{2}}(\mathbf{r}) a_{j_{2}}\right| 0\right\rangle,
$$

where the summation is over all single-particle orbits and $\phi_{j}(\mathbf{r})$ is the corresponding wavefunction in the lowest LL. In Fig. 1, we show how $\rho(\mathbf{r})$ in the ground state evolves with disorder strength $W$ for the number of electrons $N_{e}=10$ in the $N=2$ LL. The aspect ratio of the system is $A=b / a$ $=0.75$, which is an optimized geometry for observing the instability toward the formation of a stripe phase as suggested by RHY. For a very weak disorder strength, $W$ $=0.015$, the typical behavior of $\rho(\mathbf{r})$ is shown in Fig. 1(a) as a function of the two-dimensional coordinates. Two nearly perfect stripes are formed along the $y$ direction with essentially no density modulations along the stripes. This is, to the best of our knowledge, the first direct demonstration by an exact method of the stripe ordered ground state in finite systems in the presence of weak disorder.

It is important to note that in the clean case studied by RHY, the ground state and all exact many-body eigenstates are spatially homogeneous due to the translational symmetry of the Hamiltonian. Only certain combinations of the five low-lying, nearly degenerate eigenstates, separated by the unique wave vector $\mathbf{q}^{*}$, can give rise to a stripe phase that has a substantial overlap with the HF unidirectional CDW state. It may be possible to realize such a constructed stripe phase in the thermodynamic limit in clean systems if an external potential modulation is present with a wave vector that matches $\mathbf{q}^{*}{ }^{8}$ However, when a relatively weak disorder is turned on, it causes a natural mixing of the low-lying eigenstates and the near degeneracy is lifted. At the same time, the random disorder potential necessarily introduces an oscillatory component matching the wave vector $\mathbf{q}^{*}$. The presence
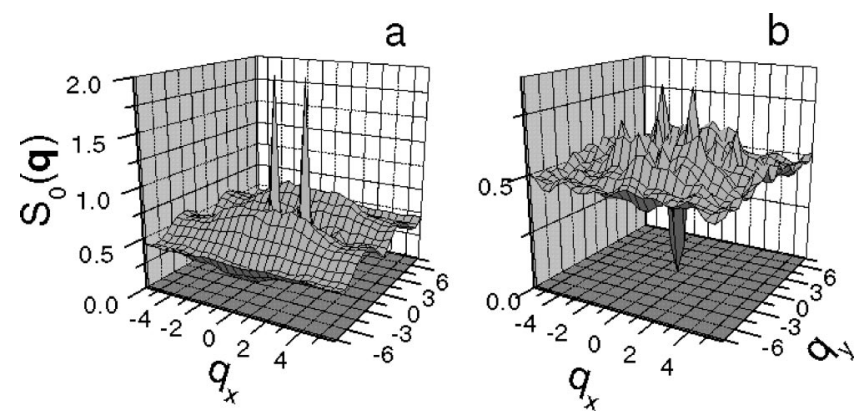

FIG. 2. The static density-density correlation function $S_{0}(\mathbf{q})$ at (a) weak disorder $W=0.09<W_{c}$ and (b) strong disorder $W=0.14$ $>W_{c}$.

of the enormous static density response $\chi\left(\mathbf{q} \rightarrow \mathbf{q}^{*}\right)$ then gives rise to the unidirectional $\mathrm{CDW}$, or the stripe order in the ground state that we observe.

To further support the picture of weak disorder induced stripe order in the ground state, we calculated the projected equal time density-density correlation function $S_{0}(\mathbf{q})$ defined as follows:

$$
S_{0}(\mathbf{q})=\frac{1}{N_{e}}\left\langle 0\left|\sum_{i, j} e^{i \mathbf{q} \cdot\left(\mathbf{R}_{i}-\mathbf{R}_{j}\right)}\right| 0\right\rangle .
$$

We find that for weak disorder of strength $W \leqslant 0.05, S_{0}(\mathbf{q})$ is quantitatively indistinguishable from the result of RHY for the corresponding clean system, showing strong and sharp peaks at $\mathbf{q}^{*}=( \pm 0.97,0)$ for $2 \pi / a=0.485$, with a peak value $S_{0}\left(\mathbf{q}^{*}\right)=3.7$ in the case of $N_{e}=10$. The peak value of $S_{0}(\mathbf{q})$ increases with the number of electrons to 4.9 at $N_{e}=12$. It can be seen from Fig. 1(a) that the width of the stripes is given by $D_{s}=a / 2$, which equals $2 \pi / q_{x}^{*}$ with $q_{x}=4 \pi / a$, as implied in this picture. It is interesting to note that the edges of the stripes are rather soft, resembling that of a single dominant harmonic variation. Next, we discuss the evolution of the ground state with increasing disorder strength $W$. Figure 1(b) shows the real space profile of $\rho(\mathbf{r})$ at $W=0.045$. There are still two complete stripes with the same stripe width $D_{s}$. Although density modulations along the stripes are still minimal, the shape of the stripes exhibits pronounced variations in the stripe direction. Thus we conclude that for weak to moderate disorder strength, the ground state is in the quantum Hall smectic phase. ${ }^{2,12,13}$

The behavior of the static density-density correlation function $S_{0}(\mathbf{q})$ in the smectic phase is exemplified in Fig. 2 (a) for $W=0.09$. Despite the relatively strong disorder potential, the peaks of $S_{0}(\mathbf{q})$ are still locked at $\mathbf{q}^{*}$, but the peak value has been reduced to 1.96. The entire structure of $S_{0}(\mathbf{q})$ remains qualitatively unchanged from the clean limit. ${ }^{8}$ The absence of emerging peaks at other $\mathbf{q}$ suggests that the smectic ground state is stable against density modulations along the stripes, even in the presence of translational-symmetry breaking disorder potentials.

We next demonstrate that further increasing the disorder strength $W$ eventually makes the smectic phase unstable. The projected density $\rho(\mathbf{r})$ is shown in Fig. 1 (c) for $W=0.1$. At this value of $W$, the smectic phase is on the brink of being 
unstable. $\rho(\mathbf{r})$ shows very strong shape fluctuations and significant density modulations along the stripes with detectable interstripe correlations. At a still larger $W=0.14$, the $\rho(\mathbf{r})$ plotted in Fig. 1(d) shows that the long-range stripe order is lost: the stripes become raptured and riddled with defects, very much like in the nematic phase. Furthermore, the local orientation of the short-range stripes show indications that it has been rotated away from the $y$ axis. The corresponding $S_{0}(\mathbf{q})$ is shown in Fig. 1(b). It is clear that the primary peaks around $\mathbf{q}^{*}$ become very much broadened with significantly reduced peak values. Concomitantly, several competing peaks appear in $S_{0}(\mathbf{q})$, marking the breakdown of the smectic phase by random disorder and the emergence of a nematic/isotropic phase with local electron-density variations. The positions of these peaks are random and dependent on the disorder configuration. These results suggest that there exists a smectic to nematiclike or isotropic phase transition, or a sharp crossover that takes place at a critical/ characteristic $W_{c}$ between $W=0.10$ and $W=0.14$. We note that it is somewhat difficult to distinguish between a nematiclike and an isotropic phase with short-range stripe correlations in our finite-size systems.

In order to pin down $W_{c}, 40$ different disorder configurations have been studied in detail. We find that the disorder strength $W_{c}$, at which multiple random peaks develop in $S_{0}(\mathbf{q})$ and compete with the primary peaks at $\mathbf{q}^{*}$, is always around a narrow region of $W_{c}=0.12$. As expected, the same value of $W_{c}$ can be extracted directly from the spatial distributions of $\rho(\mathbf{r})$ shown in Fig. 1. In Fig. 3(a), we plot the disorder averaged peak values of $S_{0}\left(\mathbf{q}^{*}\right)$ for $N_{e}=8$ and $N_{e}$ $=10$ as a function of $W . S_{0}\left(\mathbf{q}^{*}\right)$ stays almost constant for relatively weak disorder $(W \leqslant 0.05)$. Then it begins to drop rather quickly around $W=0.07$, while the strong peaks of $S_{0}(\mathbf{q})$ remain at $\mathbf{q}^{*}$ and the emergent peaks at other values of $\mathbf{q}$ are absent. For even stronger disorder, $S_{0}\left(\mathbf{q}^{*}\right)$ becomes comparable to the background value of $S_{0}(\mathbf{q})$, which is approximately 0.5 for electrons at half filling, and simultaneously random peaks emerge around $\mathbf{q}^{*}$. Such a change occurs around $W_{c}=0.12$ for $N_{e}=10$ as marked in Fig. 3(a). Due to the finite-size effect, the value of $W_{c}$ for $N_{e}=8$ is around 0.1 .

We next construct the "order parameters" of the smectic phase $\Delta_{x}$ and $\Delta_{y}$, which essentially describe the overlap of the true ground state with the HF unidirectional CDW state, ${ }^{8}$

$$
\Delta_{x}=\left\langle\left[\sum_{j_{x}} \frac{1}{n_{s}}\left(n_{j_{x}}-n_{j_{x}+j 0}\right)^{2}\right]^{1 / 2}\right\rangle .
$$

Here, $\langle\cdots\rangle$ denotes the average over 200 and 40 disorder configurations for $N_{e}=8$ and $N_{e}=10$, respectively. In Eq. (4), the summation is over all single-particle cyclotron orbits along $x$ direction with a density $n_{j_{x}}=\left\langle 0\left|a_{j_{x}}^{+} a_{j_{x}}\right| 0\right\rangle, n_{s}$ $=2 N_{e}$ is the number of orbits in each LL whereas $j_{0}$ $=\left(D_{s} / 2\right) /\left(a / n_{s}\right)=n_{s} / 4$ is the half-stripe width in units of orbit number. $\Delta_{y}$ can be similarly defined.

In Fig. 3(b), both $\Delta_{x}$ and $\Delta_{y}$ are shown as a function of the disorder strength $W$. At $W=0$, the ground state is in the uniform phase for the translational invariance of the system,
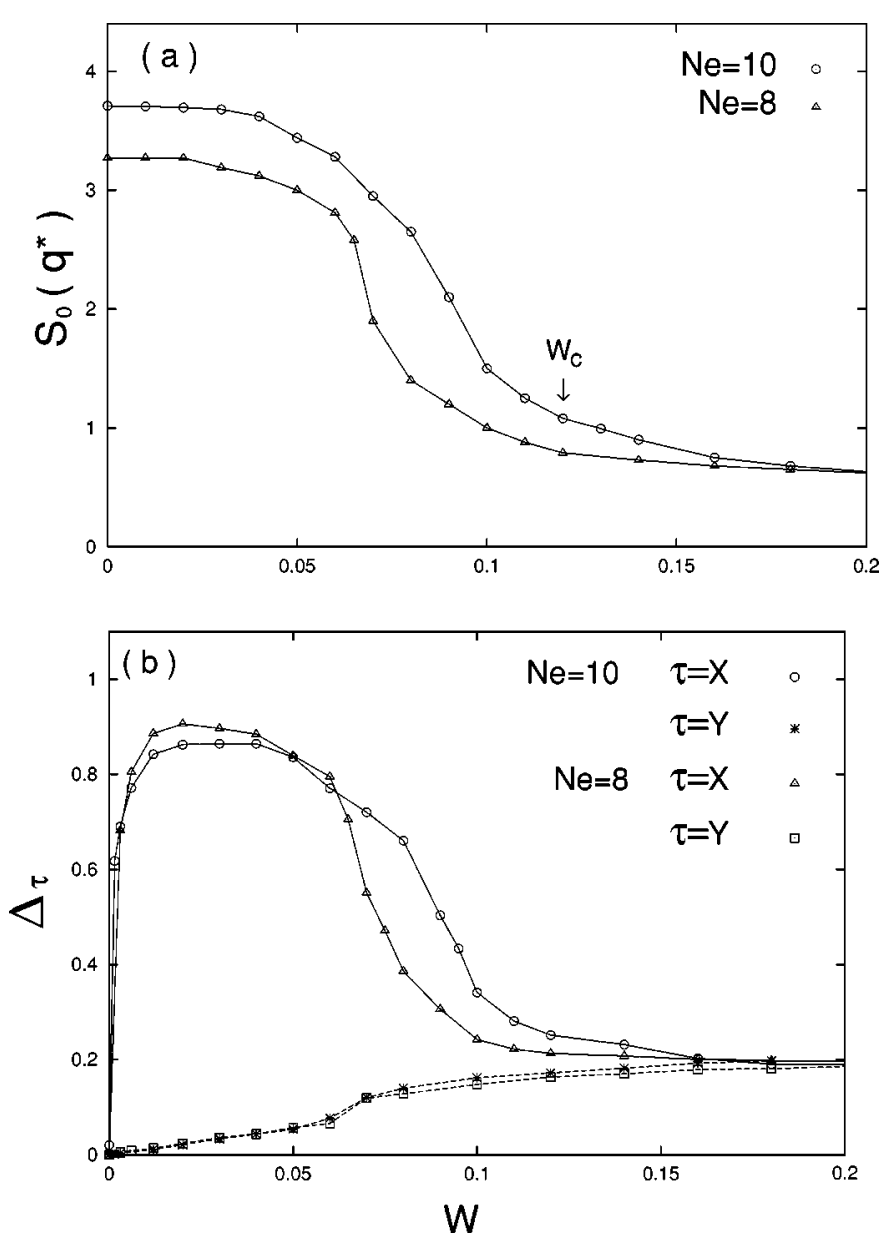

FIG. 3. (a) The peak value of the $S_{0}(\mathbf{q})$ at the primary wave vectors $\mathbf{q}=\mathbf{q}^{*}$ as a function of $W$ with a critical value $W_{c} \simeq 0.12$. (b) The constructed "order parameters" of the smectic phase $\Delta_{x}$ and $\Delta_{y}$ as a function of $W$.

$\Delta_{x}=\Delta_{y}=0$. However, when we switch on a weak disorder potential that stabilizes the stripes stacked in the $x$ direction, $\Delta_{x}$ becomes much larger than $\Delta_{y}$ and is close to 1 . Only for the HF wave function, where the cyclotron orbits are either fully occupied or completely empty, $\Delta_{x}^{\mathrm{HF}}=1$. The deviation of $\Delta_{x}$ from 1 at small $W$ is due to the smooth variation of $\left\langle 0\left|a_{j}^{+} a_{j}\right| 0\right\rangle$ between high and low occupation orbits, i.e., the softness of the edges shown in $\rho(\mathbf{r})$. This remains true for $N_{e}=12$. Although larger system sizes are needed to rule out the finite-size effect, we point out that the absence of the sharp edges is an important observation since many of the recent theoretical descriptions of the smectic phase ${ }^{2-5}$ share a common starting point of coupled one-dimensional edge states along the stripes, which requires the presence of sharp edges.

When $W$ is increased to $0.1, \Delta_{x}$ drops quickly to about 0.3 , corresponding to the development of the local density modulations along the stripes shown in Fig. 1(c). Again we see that $W_{c} \simeq 0.12$ can be considered as a transition point between a smectic and an isotropic phase with comparable $\Delta_{x}$ and $\Delta_{y}$, signaling the loss of long-range anisotropic charge ordering in the ground state. In the strong $W$ region, both $\Delta_{x}$ and $\Delta_{y}$ effectively describe the strong local chargedensity fluctuations. 


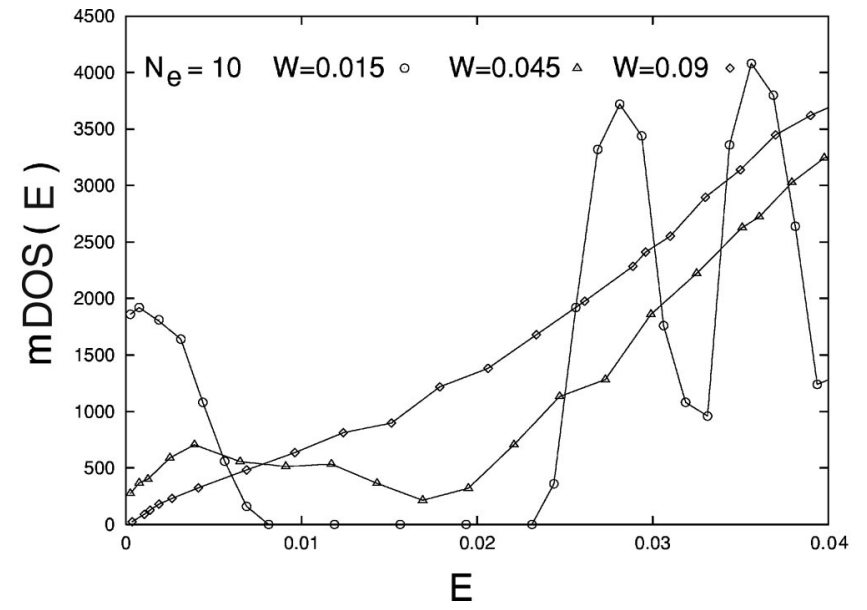

FIG. 4. The mDOS versus energy $E$ at three different values of $W$ for $N_{e}=10$ and $N=2$.

To develop further insights into the physical origin and the nature of such a smectic, quantum Hall liquid state, we plot, in Fig. 4, the mDOS for $N_{e}=10$ in the second LL. It is clear that for weak disorder, represented by $W=0.015$, the $\mathrm{mDOS}$ exhibits features of a finite density of in-gap states. Specifically, there is a finite density of low-lying states separated from all excited states by a finite energy gap. This is a direct evidence that the ground state is compressible. The gap value is $E_{g} \simeq 0.015$ for both $N_{e}=10$ and $N_{e}=8$. All states below the gap (its number equals $N_{e}$ ) have a similar $S_{0}(\mathbf{q})$ and projected electron density $\rho(\mathbf{r})$, each represents a stripe state in the $y$ direction shifted away by one orbit distance. They correspond to long-wavelength phonon excitations. The higher-energy states above the gap are isotropic, corresponding to interstitial and defectlike excitations accommodated by the impurity potential. The presence of such a finite-energy gap in weak disorder and the nature of the in-gap states suggest that the smectic state obtained here is likely to be the stable ground state in the thermodynamic limit.

Upon increasing $W$, the isotropic states move toward lower energy and the gap is reduced. At $W=0.045$, the gap region has turned into a minimum in the mDOS. However, the mDOS remains finite in the limit $E \rightarrow 0$, although its value has been reduced as states are transfered to higher energies by disorder. The filling of the energy gap begins to have an impact on the projected electron density in the ground state shown in Fig. 1(b) in the form of pronounced shape fluctuations along the stripe directions. With the further increase of $W$, the gap eventually collapses. At $W$ $=0.09$, the $\operatorname{mDOS}$ as a function of $E$ exhibits a remarkable behavior: it vanishes linearly in the limit $E \rightarrow 0$. This behavior remains qualitatively unchanged in the strong $W$ region and is insensitive to sample sizes for $N_{e}=8,10$, and 12 . Further increase of $W$ in the absence of the spectral gap causes strong mixing of the low-lying and the excited states and eventually destroys the smectic phase in favor of an isotropic phase with short-range stripe correlations.

We have also studied the transport property of the anisotropic CDW state by calculating the Thouless energy $\Delta E_{x x}$ or $\Delta E_{y y}$, which is the change in the ground-state energy under the change of the boundary condition in the $x$ or $y$ direction from periodic to antiperiodic. We find that for weak disorder, e.g., $W=0.015, \Delta E_{y y}$ is about 50 times bigger than $\Delta E_{x x}$, suggesting ${ }^{14}$ a large transport anisotropy associated with the quantum Hall smectic phase consistent with the experimental findings.

We would like to acknowledge helpful discussions with M. Fogler, E. Rezayi, and M. P. A. Fisher. D.N.S. would like to thank the hospitality of the Aspen Center for Physics where this work was initiated. D.N.S. was supported by Petroleum Research Fund Grant No. ACS-PRF \# 36965-AC5 and Research Corporation Award No. CC5643. Z.W. was supported by DOE Grant No. DE-FG02-99ER45747 and an award from Research Corporation.
${ }^{1}$ M.P. Lilly, K.B. Cooper, J.P. Eisenstein, L.N. Pfeiffer, and K.W. West, Phys. Rev. Lett. 82, 394 (1999); R.R. Du, D.C. Tsui, H.L. Stormer, L.N. Pfeiffer, K.W. Baldwin, and K.W. West, Solid State Commun. 109, 389 (1999).

${ }^{2}$ E. Fradkin and S.A. Kivelson, Phys. Rev. B 59, 8065 (1999).

${ }^{3}$ A.H. MacDonald and M.P.A. Fisher, Phys. Rev. B 61, 5724 (2000).

${ }^{4}$ H.A. Fertig, Phys. Rev. Lett. 82, 3693 (1999).

${ }^{5}$ A. Lopatnikova, B.I. Haperin, S.H. Simon, and X.-G. Wen, cond-mat/0105079 (unpublished).

${ }^{6}$ D.G. Barci, E. Fradkin, S.A. Kivelson, and V. Oganesyan, Phys. Rev. B 65, 245319 (2002).

${ }^{7}$ A.A. Koulakov, M.M. Fogler, and B.I. Shklovskii, Phys. Rev. Lett. 76, 499 (1996); R. Moessner and J.T. Chalker, Phys. Rev. B 54, 5006 (1996).

${ }^{8}$ E.H. Rezayi, F.D.M. Haldane, and K. Yang, Phys. Rev. Lett. 83,
1219 (1999); 85, 5396 (2000).

${ }^{9}$ Note that the melting temperature of the stripes in the HartreeFock theory vanishes at a critical disorder strength, T.D. Stanescu, I. Martin, and P. Phillips, Phys. Rev. Lett. 84, 1288 (2000).

${ }^{10}$ All results remain essentially unchanged under antiperiodic boundary conditions.

${ }^{11}$ For reviews, see, e.g., The Quantum Hall Effect, edited by R. E. Prange and S. M. Girvin, 2nd ed. (Springer, New York, 1990).

${ }^{12}$ D.G. Barci and E. Fradkin, Phys. Rev. B 65, 245320 (2002).

${ }^{13}$ M.M. Fogler, cond-mat/0111001 (unpublished).

${ }^{14}$ For a noninteracting system, Thouless energy $\Delta E_{x x}$ or $\Delta E_{y y}$ can be related to the conductance quantitatively. For an interacting system, $\sigma_{x x}$ should still be a monotonic function of $\Delta E_{x x}$ as it measures the general extensiveness of the wave function by its sensitivity to the change in the boundary condition. 